\title{
Influence of Vitamin D deficiency and Breast Cancer on incidence of periodontal diseases in females
}

\author{
Haseena Fuad ${ }^{1}$, Ebtissam Mohammed Al Madi ${ }^{2}$, Fuad Latheef ${ }^{3}$ \\ ${ }^{I}$ (Department of Preventive Dentistry, College of Dentistry, Princess Nora \\ BintAbdulrahmanUniversity, Riyadh, Saudi Arabia. \\ ${ }^{2}$ (Dean, College of Dentistry, Princess Nora BintAbdulrahmanUniversity, Riyadh, Saudi Arabia.) \\ ${ }^{3}$ (Department of Prosthodontics, East Riyadh Dental specialty Centre, Riyadh, Saudi Arabia)
}

\begin{abstract}
Periodontal disease is an infectious disease caused by bacterial infection and can be treated and prevented by the elimination of bacterial plaque in the adequately nourished individual. Vitamin $D$ is crucial for systemic health; nevertheless, its deficiency is highly prevalent, present in 30-50\% of the general population. Evidence has demonstrated that vitamin D deficiency may place subjects at risk for not only infectious and chronic inflammatory diseases like periodontitis but also breast cancer. Through its effect on bone and mineral metabolism, innate immunity, and several vitamin $D$ receptor gene polymorphisms, vitamin $D$ has been reported to be associated with the periodontal disease.

Historically, periodontal disease was seen as an inflammatory disease with little link to nutrition, and many early epidemiological studies failed to show associations between nutritional status and periodontal disease. This paper systematically reviewed published literature on possible associations between different nutrient deficiencies, periodontal disease and breast cancer. Overall, the studies included were limited due to restricted selection and information bias.
\end{abstract}

Keywords: Anti-inflammatory, Breastcancer, ClinicalAttachmentLoss, Periodontaldisease, VitaminD deficiency.

\section{Introduction}

Periodontal disease is a multifactorial disease initiated by a bacterial infection leading to a response by the host. Periodontal disease involves complex interactions of host defense, bacteria, and virus. Periodontal disease is initiated by a biofilm of bacteria on the teeth which triggers an immune-inflammatory response in the adjacent host tissues. [1] Numerous oral bacterial species have been associated with periodontal disease.[2]Hallmarks of this disease are bone loss and an inflammatory, immune reaction.

Vitamin D plays a role in both calcium and bone homeostasis as well as in the immune function. Vitamin D and calcium deficiencies lead to bone loss and increased inflammation, both well-known symptoms of periodontal disease.Susceptibility to periodontal disease varies among patients as displayed by their onset, extent, and severity of the disease.[3]

Carcinogenesis has been described as "a multistep process and these steps reflect genetic alterations that drive the progressive transformation of normal human cells into highly malignant derivatives".[4] A history of low degree of chronic inflammation, such as that seen in periodontal disease, is involved in carcinogenesis, and that the incidence of breast cancer would be associated with periodontal disease and characteristic tooth loss. A few earlier studies have reported an association between periodontitis and cancer.[5]

The relationship between the coincidence oftheoccurrence of tumors at particular latitudes andthe vitamin D deficiency has for many years beenthe subject of numerous research studies. The discoverythat most tissues have receptors for the vitaminD was a breakthrough in understanding therole of the vitamin $\mathrm{D}$ in the mechanism of tumor development. The research showed that the vitaminD, by increasing the tissue differentiation andpromoting the apoptosis, reduces the formation of metastases and the angiogenesis.[6] The presence of different alleles for the VDR gene is responsiblefor a different risk of tumor formation.

\section{Etiology Of Periodontal Diseases}

Periodontal diseases are a series of complex, distinct, pathologic entities caused by the interaction of bacterial plaque and the host. This interaction results in destruction of the supporting alveolar bone and connective tissue. Periodontal disease, as typified by periodontitis, has a varied and highly complex polymicrobial etiology. Sub gingival (below the gum-line) plaque within diseased periodontal sites is typically enriched in anaerobic and proteolytic species of bacteria.[7]

Periodontitis is a chronic inflammatory disease of the supporting structures of the teeth initiated by pathogenic bacteria, predominantly porphyromonas gingivalis, tannerella forsythensis and treponima denticola, associated with numerous others in plaque biofilm attached to root surfaces, leading to deepening periodontal pockets and alveolar bone destruction.[8] 
Although bacterial plaque has been implicated as the primary etiologic agent in most forms of periodontal disease, there are local and systemic factors which may modify both microbial and host components. Local factors may favor plaque accumulation and maturation, while systemic factors may modulate and decrease the host's protective response.[9]

\section{Potential role of vitamin D on periodontal disease}

\subsection{Deficiency of vitamin D on skeletal homeostasis and its effects on periodontium}

Since 1865, it is known that low solar ultraviolet-B (UVB) doses have been associated with increased risk of tooth loss.[10] A role of vitamin D in reducing risk of dental caries was known in 1928 [11] and, by 1937, increased solar Ultra violet-B ray exposure was associated with reduced risk of dental caries [12].

Vitamin D and calcium are fundamental for bone mineralization and for the prevention of osteoporosis. Vitamin D plays an important role in calcium homeostasis, promoting calcium absorption in the intestine and stimulating osteoblasts to enable normal bone growth and preservation. Severe vitamin D deficiency causes mineralization defects (osteomalacia), but chronically low intake of vitamin D and calcium leads to negative calcium balance and bone loss, and it is reasonable to expect this effect to occur in alveolar bone as it does in other bones of the body. A number of epidemiological studies have reported a positive association between low bone mass or osteoporosis and alveolar bone loss and tooth loss.[13,14]This suggests that low bone mass is a risk factor for the development and progression of periodontal disease.[15]

In a recent study Jabbar $S$ et al [16]found that periodontal disease is more common in women with osteoporosis and is associated with lower vitamin D and higher RANKL(Receptor activator of nuclear factor kappa-B ligand ) and osteoprotegerin.

\subsection{Vitamin D , its anti- inflammatory and anti-microbial properties.}

In addition to its action on skeletal homeostasis, vitamin D and in particular its hormonally active form, 1 $\alpha, 25$-dihydroxyvitamin D has anti-inflammatory and anti-microbial effects, via modulation of inflammatory cytokine production by immune cells, and stimulated secretion of peptides with anti-bacterial action by cells of the monocyte-macrophage lineage.[17]

Vitamin D deficiency has also been linked to periodontal disease risks.[18,19]The likely mechanisms by which vitamin D reduces risks of dental caries and periodontal disease include induction of cathelicidin and defensins, which have antibacterial properties.[20]Cathelicidins and defensins are small peptides with amphipathic structures that allow them to disrupt the integrity of the pathogen cell membrane, resulting in its death. Vitamin D modulates T-cell responses and has anti-inflammatory properties, but boosts innate immune responses by induction of the human gene for cathelicidin.[21]Periodontitis manifested as higher attachment loss, was found to be associated with lower serum $1,25(0 \mathrm{H}) \mathrm{D}$, concentrations, which may be explained by the anti-inflammatory effects of vitamin D.[22] In the treatment of periodontal disease, vitamin D may be of benefit not only for its direct effect on bone metabolism but also due to its anti-inflammatory and cAMP(Cyclic adenosine monophosphate)-stimulating properties.[23]

These multiple actions of vitamin D are potentially appealing for the management of patients with periodontal disease, whose pathogenesis is based on chronic bacterial-driven inflammation. The inflammatory response leads to tissue destruction either by direct action of bacterial products, or by activation of host defense cells and secretion of inflammatory mediators. These locally produced factors eventually result in connectivetissue breakdown and bone loss via activation of osteoclast mediated bone resorption.[24,25]

Indeed, vitamin D deficiency may be linked to increased risk of infectious diseases. This suggests that vitamin D may be of benefit in the treatment of periodontitis, not only because of its direct effects on bone metabolism, but also because it may have antibiotic effects on periodontopathogens and inhibit inflammatory mediators that contribute to the periodontal destruction.

\subsection{Effects of vitamin D supplementation on periodontal health}

Calcium and vitamin D supplementation has a modest positive effect on periodontal health, and consistent dental care improves clinical parameters of periodontal disease regardless of such supplements. The findings of recent studies raise the possibility that vitamin D, perhaps at higher doses, may positively impact on periodontal disease severity. Calcium and vitamin D supplementation may reduce the severity of periodontal disease if used at doses higher than 800-1,000 IU daily and supported the rationale for testing the potential beneficial role of vitamin $\mathrm{D}$ on periodontal disease in randomized clinical trials. $[17,26]$

The significant role of a proper diet is emphasized in the prevention of periodontal diseases. The dietary supplementation with calcium and vitamin D may improve periodontal health, increase bone mineral density in the mandible and inhibit alveolar bone resorption.[27] Therefore, administering vitamin D seems advisable in order to increase its level in patients with the periodontal diseases. 
Although the number of studies on the effects of calcium or vitamin D intake on oral outcomes is limited, they suggest that higher intake levels are associated with reduced prevalence of clinical attachment loss and lower risk of tooth loss.[28]

It is reasonable to think that calcium and vitamin D affect oral bone in the same way that they affect other bones of the body, and various studies, including analyses of data from NHANES III, have found associations between periodontal health and intakes of calcium and vitamin D. Inadequate intake of vitamin D could increase levels of periodontal disease either through an effect on bone mineral density or a suppression of vitamin D's immunomodulatory effects. [29,30]

\subsection{Vitamin D receptor polymorphism and periodontitis}

Several VDR(vitamin D receptor) restriction fragment length polymorphism (RFLPs) associate with several diseases namely secondary hyperparathyroidism in the renal failure, osteoporosis, cancer, nephrolithiasis, diabetes, and periodontal disease .[31]

The BsmI RFLP in combination with other RFLPs are associated with early onset periodontitis and chronic periodontitis in Korean population. The ApaI, BsmI and FokIRFLPs( restriction fragment length polymorphism) have been reported to confer elevated risk of severe chronic periodontitis in Japanese men. These RFLPs are associated with bone and mineral disease and the TaqI and FokI RFLPs are associated with increased cancer risk, such as prostate and breast malignancy.[32]These findings indicate that $1,25(\mathrm{OH}) 2 \mathrm{D} 3$ plays a role in prevention of periodontal disease and that hypomorphic VDR alleles and reduced levels of 1,25(OH)2D3 - I,25dihydroxy vitamin D3 may be associated with the periodontal disease.[33]

\section{Relationship between periodontal disease and breast cancer}

An additional benefit of vitamin $\mathrm{D}$ is reduced inflammation, primarily through modifying the production of cytokines from pro- to anti-inflammatoryfactors [34] and inflammation is a risk factor for breast cancer . [35]

4.1.Connection between chronic inflammation and cancer incidence

The connection between inflammation and cancer has been suggested as consisting of 2 mechanisms: extrinsic and intrinsic mechanisms. In the extrinsic mechanism, a chronic inflammatory state increases the risk of cancer while in the intrinsic mechanism, acquired genetic alterations trigger tumor development.

Chronic infection may stimulate the formation of epithelial-derived tumors through an indirect mechanism involving activation of surrounding inflammatory cells. It may also expose epithelial cells to mutagens. Microorganisms associated with the inflammatory process as well as their products can activate host cells such as inflammatory cells, fibroblasts, and epithelial cells to generate a variety of substances which can induce DNA damage in epithelial cells. Chronic inflammatory processes are frequently associated with the release of large amounts of cytokines, chemokines, growth factors, and other signals that provide an environment for cell survival, proliferation, migration, angiogenesis, and inhibition of apoptosis. This environment may help epithelial cells to accumulate mutations and drive these mutant epithelial cells to proliferate, migrate, and give them a growth advantage.[36]

\subsection{Periodontitis and incidence of breast cancer}

An epidemiological study shows that, fairly young individuals with periodontal disease and missing molars are at increased risk of premature death caused by life threatening diseases such as malignant neoplasm, cardiovascular diseases, and diseases of the digestive system. [37]

Later the same authors found a statistically significant correlation between periodontal disease, related tooth loss and incidence of breast cancer. Periodontal disease with any missing molar teeth was associated with nearly a 12-fold increased risk of breast cancer than no missing molars. It was suggested that the bacterial infection associated with periodontal disease was the risk factor for breast cancer through effects of infection and inflammation.[38]

Due to poor oral hygiene and gram- negative anaerobic bacterial infection, chronic periodontitis is closely associated with human cytomegalovirus and Epstein- Barr virus co- infection. It is supposed that these viruses act together to control immune response to bacterial challenges; these viruses and bacteria act together to lead to low degree chronic inflammation and carcinogenesis. Periodontal disease is associated with an increased production of reactive oxygen species which, if not buffered sufficiently, cause damage to the host cells and tissues. The byproducts of the oral inflammation enter the bloodstream, which may enhance cellular proliferation and mutagenesis, allowing for the development and spread of cancer.[39] 


\section{Conclusion}

The interaction between oral and systemic health are bidirectionaland complex, involving many pathways.Although classically thought of as a "bone hormone,"vitamin D plays a role in other parts ofthe body. It is a predictorfor bone health but is also a potential independent predictor ofrisk for cancer and other chronic diseases like periodontitis. Oral infections, in particular periodontal disease, triggers inflammatory reactions and detrimentally affect systemichealth where inflammations are known to play a role in the pathogenesis, namely cancer.

To further determine a possible cause-andeffectrelationship there needs to be a randomized control trial exploring supplementation of vitamin D with periodontal disease measures. Until then, the relationship between vitamin $\mathrm{D}$ and periodontitis remains unknown. Research on influence of a chronic disease like periodontitis on breast cancer is verymuch needed, especially with the growing knowledge thatoral and systemic health is linked. Current research todefine these risks is scant. As we gain a greater understanding of the linkage betweenoral and systemic health, the need for closer collaborationbetween dental and medical professionals is important forthe care of patients with breast cancer.

\section{Acknowledgement}

Authors are thankful to Dr. Ebtissam Al madi, Dean, College of Dentistry, Princess Nora University and to Princess Nora Bint Abdulrahman University for their support for research.

\section{References}

[1] Page RC, Offenbacher S, Schroeder HE, Seymour GJ, Kornman KS, Advances in the pathogenesis of periodontitis: summary of developments, clinical implications and future directions, Periodontol 2000, 14,1997,216-248.

[2] Haffajee AD, SocranskySS ,Microbiology of periodontal diseases: introduction, Periodontol 2000, 38,2005,9-12

[3] Hildebolt CF, Effect of vitamin D and calcium on periodontitis,J of Periodontol, 76(9),2005,1576-1587.

[4] Hanahan D, Weinberg RA, The hallmarks of cancer,Cell ,100,2000, 57-70.

[5] Michaud D, Joshipura K, Giovannucci E, Fuchs CS, A prospective study of periodontal disease and pancreatic cancer in US male health

professionals, J Natl Cancer Inst 99,2007, 171-175.

[6] Van den Bemd GJ, Chang GT, Vitamin D and vitamin D analogs in cancer treatment, Current Drug.Targets,3,2002,85-94.

[7] Rylev M, Kilian M, Prevalence and distribution of principal periodontal pathogens worldwide. Journal of Clinical Periodontology, 35(8Suppl),2008,346-361.

[8] Page RC, KornmanKS, The pathogenesis of human periodontitis: an introduction, Periodontology 2000,14,1997,9-11.

[9] Socransky SS, Haffajee AD, Periodontal microbial ecology, Periodontology 2000, 38,2005,135-187.

[10] Lewis JR, Exemptions from military service on account of loss of teeth, Dental Cosmos ,7,1865,240-242.

[11] Mellanby M, Pattison CL, The action of vitamin D in preventing the spread and promoting the arrest of caries in children,British MedicalJournal,2,1928,1079-1082.

[12] Mills CA, Factors affecting the incidence of dental caries in population groups, Journal of Dental Research,16,1937,417-430

[13] HildeboltCF,Osteoporosis and oral bone loss,Dentomaxillofacial Radiology,26,1997,3-15.

[14] Jeffcoat MK, Reddy MS, Alveolar bone loss and osteoporosis: Evidence for a common mode of therapy using the bisphosphonate alendronate, in Z Davidovitch ,LA Norton (Ed.), Biological Mechanisms of Tooth Movement and Craniofacial Adaptation, 1(Cambridge, MA: Harvard Society for the Advancement of Orthodontics, 1996) 365-373.

[15] ]Hildebolt CF, Effect of vitamin D and calcium on periodontitis, Journal of Periodontolgy,76(9),2005,1576-1587

[16] Jabbar S, Drury J, Fordham J, Plasma vitamin D and cytokines in periodontal disease and postmenopausal osteoporosis, Journal of Periodontal Research, 46(1),2011,97-104.

[17] M. Nathalia Garcia, One-year Effects of Vitamin D and Calcium Supplementation on Chronic Periodontitis, Journal of Periodontology, 82(1),2011,25-32.

[18] Amano Y, Komiyama K, Makishima M, Vitamin D and periodontal disease, Journal of Oral Science,51(1),2009,11-20.

[19] Grant WB, Boucher BJ , Are Hill's criteria for causality satisfied for vitamin D and periodontal disease? Dermato Endocrinology, 2(1),2010,30-36.

[20] Gombart AF, The vitamin D-antimicrobial peptide pathway and its role in protection against infection, Future Microbiology, 4(9),2009,1151-1165.

[21] Hewison M, Antibacterial effects of vitamin D, Nature Reviews Endocrinology, 7(6),2011,337-45.

[22] Krall EA, Wehler C, Garcia R1, Calcium and vitamin D supplements reduce tooth loss in the elderly,American Journal of Medicine, 11(6),2001,452-456.

[23] Stein SH, Tipton DA, Vitamin D and its impact on Oral Health - An Update, Journal of Tennessee Dental Association, 91(2),2011,30-33

[24] Page RC, Eke PI,Case definitions for use in population-based surveillance of periodontitis, Journal of Periodontology,78,2007,1387-1399.

[25] Cochran DL, Inflammation and bone loss in periodontal disease. Journal of Periodontology,79,2008,1569-1576.

[26] Miley DD, Garcia MN, Hildebolt CF, Cross-sectional study of vitamin D and calcium supplementation effects on chronic periodontitis, Journal of Periodontology,80(9),2009,1433-1439.

[27] Hildebolt CF, Pilgram TK, Dotson M, Armamento-Villareal R, Häuser J, Cohen S, Estrogen and/or calcium plus vitamin D increase mandibular bone mass, Journal of Periodontology, 75,2004, 811-816.

[28] Krall EA,The periodontal-systemic connection; implications for treatment of patients with osteoporosis and periodontal disease, Annals of Periodontology, 6(1),2001,209-213.

[29] Nishida M, Grossi S G, Dunford R G, Calcium and the risk for periodontal disease, Journal of Periodontology, 71,2000, 1057-1066.

[30] Dietrich T, Joshipura K J, Dawson-Hughes B, Bischoff-Ferrari H A, Association between serum concentrations of 25hydroxyvitaminD3 and periodontal disease in the US population,American Journal of Clinical Nutrition, 80,2004,108-113. 
[31] Holick MF, Vitamin D: Importance in the prevention of cancers, type 1 diabetes, heart disease, and osteoporosis, American Journal of Clinical Nutrition,79,2004,362-371.

[32] Hennig BJ, Parkhill JM, Chapple IL, Heasman PA, Taylor JJ,Association of a vitamin D receptor gene polymorphism with localizedearly-onset periodontal diseases, Journal of Periodontology, 70,1999,1032-1038.

[33] NithyaAnand S C, Chandrasekaran, Vitamin D and periodontal health: Current concept,Journal of Indian Society of Periodontology ,17(3), 2013,302-308.

[34] Guillot X, Semerano L, Saidenberg-Kermanac'h N, Falgarone G, BoissierMC, Vitamin D and inflammation.,Joint Bone Spine, 77(6),2010,552-557.

[35] Goldberg JE, Schwertfeger KL , Proinflammatory cytokines in breast cancer: mechanisms of action and potential targets for therapeutics Current Drug Targets, 11(9),2010,1133-1146.

[36] Tezal M, Sullivan MA, Reid ME, Marshall JR, Hyland A, Loree T, Lillis C, Hauck L, Wactawski-Wende J, Scannapieco FA, Chronic periodontitis and the risk of tongue cancer,Archives of otolaryngology-head \& neck surgery ,133,2007,450-454.

[37] SoderB,Jin LJ, Klinge B, Soder PO, Periodontitis and premature death: a 16-year longitudinal study in a Swedish urban population,Journal of Periodontal Research, 42(4),2007,361-366.

[38] So“der B, Yakob M, Meurman JH, Andersson LC, Klinge B, So“derPO,Periodontal disease may associate with breast cancer. Breast Cancer Research and Treatment,127(2),2011,497-502.

[39] Saini R. Vitamins and periodontitis. Journal of Pharmacy and Bioallied Science, 3,2011,170. 